\title{
Closed-Form Solution for a Class of Continuous-Time Algebraic Riccati Equations
}

\author{
Alejandro J. Rojas
}

\begin{abstract}
In the present paper we obtain a closed-form solution for the class of continuous-time algebraic Riccati equations (ARE) with vanishing state weight, whenever the unstable eigenvalues are distinct. The AREs in such a class solve a minimum energy control problem. The obtained closed-form solution gives insight on issues such as loss of controllability and it might also prove comparable in terms of numerical precision over current solving algorithms. Keywords: Algebraic Riccati equation; Closed-form solution; Controllability; Minimum energy control; Signal-to-noise ratio.
\end{abstract}

\section{INTRODUCTION}

Riccati equations, in particular algebraic Riccati equations (AREs), are a recurrent and important feature in many theoretical control design results, see for example [1], [2] or [3].

The area of Control over Networks, on the other hand, has been a growing topic of increased interest in recent years; see for example [4], [5] and references therein. A

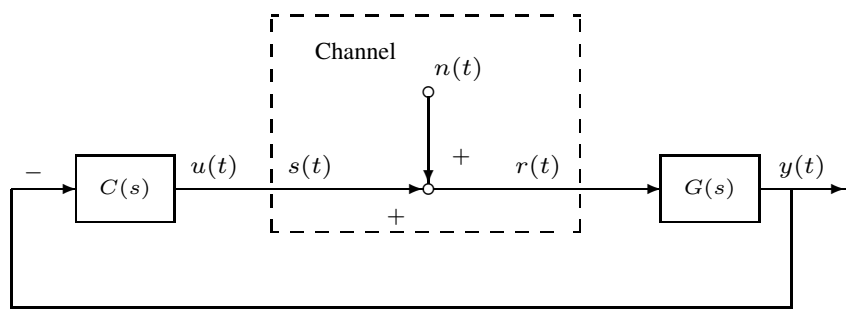

Fig. 1. General problem setting.

line of research reported in [6], [7] (and related work in [8], [9]), introduced a framework to study the fundamental limitation in stabilisability of a single-input single-output (SISO) feedback loop over channels with a signal to noise ratio (SNR) constraint.

In Figure 1 we observe the particular case of an additive white Gaussian noise (AWGN) channel located between the controller and the plant. The channel is defined by $\mathcal{P}$, the channel input power constraint

$$
\mathcal{P}>\mathcal{E}\left\{u^{2}\right\},
$$

where $\mathcal{E}$ is the expectation, and by the channel additive white Gaussian noise $n(t)$, with zero-mean and power spectral density $\Phi$. In [6] it has been proved that the AWGN channel infimal SNR for stabilisability satisfies

$$
\frac{\mathcal{P}}{\Phi}>\sum_{i=1}^{m} 2 \operatorname{Re}\left\{p_{i}\right\}
$$

Corresponding author. A.J. Rojas is with the ARC Centre of Excellence for Complex Dynamic Systems and Control, The University of Newcastle, Australia.Email: Alejandro.Rojas@newcastle.edu.au where $\operatorname{Re}\left\{p_{i}\right\}$ is the real-part of the (possibly repeated) unstable poles of the plant model $G(s)$. The same result holds for output feedback, [6, Theorem II.2], when $G(s)$ is minimum phase and for state-feedback, [6, Theorem II.1]. In the state-feedback case it is shown that the infimal SNR for stabilisability result is linked to the solution $\mathbf{P}$ of an algebraic Riccati equation (ARE)

$$
\frac{\mathcal{P}}{\Phi}>\mathbf{B}^{T} \mathbf{P B}
$$

with vanishing state weight for a state-space representation $(\mathbf{A}, \mathbf{B}, \mathbf{C}, 0)$ of $G(s)$. Both results are equivalent, suggesting that it might be possible to express $\mathbf{P}$ in closed-form.

In [7] the infimal SNR for stabilisability result is extended to include a continuous-time unstable (non)-minimum phase plant $G(s)$ with distinct unstable poles, over an additive coloured Gaussian noise (ACGN) channel with memory. For the AWGN channel (which can be seen as a particular case of an ACGN channel) [7, Theorem 1] reduces to

$$
\frac{\mathcal{P}}{\sigma^{2}}>\sum_{i=1}^{m} \sum_{j=1}^{m} \frac{r_{i} \bar{r}_{j}}{p_{i}+\bar{p}_{j}},
$$

where

$$
r_{i}=2 \operatorname{Re}\left\{p_{i}\right\} \prod_{\substack{j=1 \\ j \neq i}}^{m} \frac{p_{i}+\bar{p}_{j}}{p_{i}-p_{j}}, \forall i=1, \cdots, m .
$$

Thus, motivated by the insight that the infimal SNR for stabilisability problem can be stated as a minimum energy problem, we analyse in the present paper the class of continuous-time AREs with vanishing state weight.

The main contribution of the present paper, based on the infimal SNR for stabilisability results above, is a closed-form solution for such a class of continuous-time AREs with vanishing state weight and non repeated unstable eigenvalues. To the best knowledge of the author the closed-form solution obtained here is novel. As a result of the closed-form nature of the solution, we obtain further insights on the structure of the minimum energy problem.

The paper is organised as follows: in Section II we present the closed-form solution for the class of continuous-time AREs with vanishing state weight. We also present a series of extensions that derive from such closed-form result. In Section III we present examples and discuss the interpretation of some of the results from the previous section. Finally, in Section IV, we give concluding remarks for the present work and future directions. 
A companion to the present paper can be found in [10] where a similar analysis is proposed for the discrete-time case.

Terminology: let $\mathbb{C}$ denote the complex plane. Let $\mathbb{C}^{-}, \overline{\mathbb{C}}^{-}$, $\mathbb{C}^{+}$and $\overline{\mathbb{C}}^{+}$denote respectively the open left-plane, closed left-plane, open right-plane and closed right-plane of $\mathbb{C}$. Let $\mathbb{R}$ denote the set of real numbers, $\mathbb{R}^{+}$the set of positive real numbers, $\mathbb{R}_{o}^{+}$the set of non-negative real numbers and $\mathbb{R}^{-}$the set of real negative numbers. Let $\mathbb{Z}^{+}$denote the set of positive integers. A continuous-time signal is denoted by $x(t)$, and its Laplace transform by $X(s), s \in \mathbb{C}$. We use bold notation to represent a generic matrix A. Similarly, $\mathbf{0}$ stands for a matrix, of suitable dimensions, with all its entries set to zero and $\mathbf{I}$ for the identity matrix. Denote the element in the $i^{t h}$-row, $j^{t h}$-column of a matrix $\mathbf{A}$ as $[\mathbf{A}]_{i, j}$.

\section{Closed-Form Solution of the}

\section{Continuous-Time Algebraic RicCati Equation With VANishing State Weight}

\section{A. Assumptions}

In the present section we present a technical result which states in closed-form the solution of a class of minimum energy Riccati equations. The assumptions under consideration are

1) A minimal realisation $(\mathbf{A}, \mathbf{B}, \mathbf{C}, 0)$ of a plant such that

$$
\mathbf{A}=\left[\begin{array}{cc}
\mathbf{A}_{u} & 0 \\
0 & \mathbf{A}_{s}
\end{array}\right], \quad \mathbf{B}=\left[\begin{array}{l}
\mathbf{B}_{u} \\
\mathbf{B}_{s}
\end{array}\right], \quad \mathbf{C}=\left[\begin{array}{ll}
\mathbf{C}_{u} & \mathbf{C}_{s}
\end{array}\right],
$$

where $\mathbf{A} \in \mathbb{R}^{n \times n}, \mathbf{B} \in \mathbb{R}^{n \times 1}, \mathbf{C} \in \mathbb{R}^{1 \times n}, \mathbf{A}_{u} \in$ $\mathbb{R}^{m \times m}, \mathbf{B}_{u} \in \mathbb{R}^{m \times 1}, \mathbf{C}_{u} \in \mathbb{R}^{1 \times m}$.

2) The eigenvalues of $\mathbf{A}_{u}$ are all in $\mathbb{C}^{+}$and they are all distinct.

3) $\mathbf{A}_{u}$ is diagonal and $\mathbf{B}_{u}=\left[\begin{array}{lll}1 & \cdots & 1\end{array}\right]^{T}$.

4) The eigenvalues of $\mathbf{A}_{s}$ are all in $\overline{\mathbb{C}}^{-}$.

Notice that assumption 1) also implies that the pair $\mathbf{A}_{u}$ and $\mathbf{B}_{u}$ is controllable. Also notice that the choice of $\mathbf{A}_{u}$ and $\mathbf{B}_{u}$ in 3) is not restrictive. Indeed from 1) we have that

$$
G(s)=\underbrace{\mathbf{C}_{u}\left(s \mathbf{I}-\mathbf{A}_{u}\right)^{-1} \mathbf{B}_{u}}_{G_{u}(s)}+\underbrace{\mathbf{C}_{s}\left(s \mathbf{I}-\mathbf{A}_{s}\right)^{-1} \mathbf{B}_{s}}_{G_{s}(s)},
$$

where $G_{u}(s)$ contains all the unstable distinct poles of $G(s)$ and similarly $G_{s}(s)$ all the stable poles of $G(s)$. With the choice of $\mathbf{A}_{u}$ and $\mathbf{B}_{u}$ in Assumption 3), the coefficients of $\mathbf{C}_{u}$ can be identified from a partial fraction expansion of $G_{u}(s)$.

B. Continuous-Time Algebraic Riccati Equation with Vanishing State Weight

A continuous-time ARE is given by

$$
\mathbf{P A}+\mathbf{A}^{T} \mathbf{P}=\mathbf{P B} R^{-1} \mathbf{B}^{T} \mathbf{P}+\mathbf{Q} .
$$

In the present paper we consider a particular class of such continuous-time AREs, namely the class with vanishing state weight, that is $\mathbf{Q}=\mathbf{0}$. This is a class of AREs that we refer to as the continuous-time minimum energy ARE

$$
\mathbf{P A}+\mathbf{A}^{T} \mathbf{P}=\mathbf{P B} R^{-1} \mathbf{B}^{T} \mathbf{P} .
$$

Under the assumptions for $\mathbf{A}, \mathbf{B}$, the unique symmetric positive semi-definite solution of (2) satisfies (see [11, Lemma 2])

$$
\mathbf{P}=\left[\begin{array}{cc}
\mathbf{P}_{u} & 0 \\
0 & 0
\end{array}\right]
$$

thus reducing (2) to

$$
\mathbf{P}_{u} \mathbf{A}_{u}+\mathbf{A}_{u}^{T} \mathbf{P}_{u}=\mathbf{P}_{u} \mathbf{B}_{u} R^{-1} \mathbf{B}_{u}^{T} \mathbf{P}_{u} .
$$

\section{Main Result}

We introduce now a closed-form characterisation of $\mathbf{P}_{u}$ the non-trivial solution to the minimum energy Riccati equation in (3).

Proposition 1: (Closed-Form Solution for $R=1$ ) The closed-form solution to the minimum energy ARE in (2) with $R=1$ is given by

$$
\hat{\mathbf{P}}=\left[\begin{array}{cc}
\hat{\mathbf{P}}_{u} & \mathbf{0} \\
\mathbf{0} & \mathbf{0}
\end{array}\right]
$$

where $\hat{\mathbf{P}}_{u}$ solves (3) with $R=1$ and is given by

$$
\hat{\mathbf{P}}_{u}=\left[\begin{array}{cccc}
\frac{r_{1}^{2}}{2 p_{1}} & \frac{r_{1} r_{2}}{p_{1}+p_{2}} & \cdots & \frac{r_{1} r_{m}}{p_{1}+p_{m}} \\
\frac{r_{2} r_{1}}{p_{2}+p_{1}} & \frac{r_{2}^{2}}{2 p_{2}} & \cdots & \vdots \\
\vdots & \vdots & \ddots & \vdots \\
\frac{r_{m} r_{1}}{p_{m}+p_{1}} & \cdots & \cdots & \frac{r_{m}^{2}}{2 p_{m}}
\end{array}\right]
$$

with $r_{i}$ defined as

$$
r_{i}=2 p_{i} \prod_{\substack{j=1 \\ j \neq i}}^{m} \frac{p_{i}+p_{j}}{p_{i}-p_{j}}, \forall i=1, \cdots, m,
$$

Proof: As a first step let us recognise that matrices $\mathbf{A}_{u}$ and $\mathbf{B}_{u}$ that satisfy assumptions 2) and 3) are given by

$$
\mathbf{A}_{u}=\left[\begin{array}{cccc}
p_{1} & 0 & \cdots & 0 \\
0 & p_{2} & \cdots & 0 \\
\vdots & \vdots & \ddots & \vdots \\
0 & 0 & \cdots & p_{m}
\end{array}\right], \quad \mathbf{B}_{u}=\left[\begin{array}{c}
1 \\
1 \\
\vdots \\
1
\end{array}\right]
$$

The LHS and RHS of (3), replacing $\mathbf{P}_{u}$ as in (5), are then given by

$$
L H S=\left[\begin{array}{cccc}
r_{1}^{2} & r_{1} r_{2} & \cdots & r_{1} r_{m} \\
r_{2} r_{1} & r_{2}^{2} & \cdots & \vdots \\
\vdots & \vdots & \ddots & \vdots \\
r_{m} r_{1} & \cdots & \cdots & r_{m}^{2}
\end{array}\right]
$$


and

$$
\begin{aligned}
& R H S=\left[\begin{array}{c}
\left(\frac{r_{1}^{2}}{2 p_{1}}+\cdots+\frac{r_{1} r_{i}}{p_{1}+p_{i}}+\cdots+\frac{r_{1} r_{m}}{p_{1}+p_{m}}\right)^{2} \\
\vdots \\
\left(\frac{r_{m} r_{1}}{p_{m}+p_{1}}+\cdots+\frac{r_{m}^{2}}{2 p_{m}}\right)\left(\frac{r_{1}^{2}}{2 p_{1}}+\cdots+\frac{r_{1} r_{m}}{p_{1}+p_{m}}\right)
\end{array}\right. \\
& \left.\cdots \quad\left(\frac{r_{1}^{2}}{2 p_{1}}+\cdots+\frac{r_{1} r_{m}}{p_{1}+p_{m}}\right)\left(\frac{r_{m} r_{1}}{p_{m}+p_{1}}+\cdots+\frac{r_{m}^{2}}{2 p_{m}}\right)\right] \\
& \ddots \\
& \cdots \quad\left(\frac{r_{m} r_{1}}{p_{m}+p_{1}}+\cdots+\frac{r_{m} r_{i}}{p_{m}+p_{i}}+\cdots+\frac{r_{m}^{2}}{2 p_{m}}\right)^{2}
\end{aligned}
$$

The rest of the argument is to prove that the LHS is equal to the RHS. Consider first the elements in the diagonal for which we want to prove that $r_{i}^{2}$ is equal to

$$
\left(\frac{r_{i} r_{1}}{p_{i}+p_{1}}+\cdots+\frac{r_{i} r_{m}}{p_{i}+p_{m}}\right)^{2}=r_{i}^{2},
$$

by use of Lemma 2 in the Appendix. Next consider any element outside the main diagonal on the RHS of (3)

$$
\begin{aligned}
\left(\frac{r_{i} r_{1}}{p_{i}+p_{1}}+\cdots+\frac{r_{i} r_{m}}{p_{i}+p_{m}}\right) & \\
\left(\frac{r_{j} r_{1}}{p_{j}+p_{1}}+\cdots+\frac{r_{j} r_{m}}{p_{j}+p_{m}}\right) & =r_{i} r_{j},
\end{aligned}
$$

by again use of Lemma 2 in the Appendix. Therefore, we conclude that $\hat{\mathbf{P}}_{u}$ is indeed the closed-form solution of (3) when $R=1$, and thus $\hat{\mathbf{P}}$ is the solution of (2), which completes the proof.

\section{Extensions and Derived Results}

We now extend the result of Proposition 1 to the general case of $R=\lambda \in \mathbb{R}$.

Corollary 1: (Closed-Form Solution for $R=\lambda$ ) The closed-form solution to the minimum energy ARE in (2) with weight $R=\lambda$ is given by

$$
\hat{\mathbf{P}}^{\lambda}=\left[\begin{array}{cc}
\hat{\mathbf{P}}_{u}^{\lambda} & \mathbf{0} \\
\mathbf{0} & \mathbf{0}
\end{array}\right]
$$

where $\hat{\mathbf{P}}_{u}^{\lambda}$ solves (3) with $R=\lambda$ and is given by

$$
\hat{\mathbf{P}}_{u}^{\lambda}=\lambda\left[\begin{array}{cccc}
\frac{r_{1}^{2}}{2 p_{1}} & \frac{r_{1} r_{2}}{p_{1}+p_{2}} & \cdots & \frac{r_{1} r_{m}}{p_{1}+p_{m}} \\
\frac{r_{2} r_{1}}{p_{2}+p_{1}} & \frac{r_{2}^{2}}{2 p_{2}} & \cdots & \vdots \\
\vdots & \vdots & \ddots & \vdots \\
\frac{r_{m} r_{1}}{p_{m}+p_{1}} & \cdots & \cdots & \frac{r_{m}^{2}}{2 p_{m}}
\end{array}\right],
$$

with $r_{i}, \forall i=1, \cdots, m$, defined as in (6).

Proof: From Proposition 1 we have that $\hat{\mathbf{P}}_{u}$ satisfies the minimum energy ARE in (3) with $R=1$

$$
\hat{\mathbf{P}}_{u} \mathbf{A}_{u}+\mathbf{A}_{u}^{T} \hat{\mathbf{P}}_{u}=\hat{\mathbf{P}}_{u} \mathbf{B}_{u} \mathbf{B}_{u}^{T} \hat{\mathbf{P}}_{u} .
$$

which, since $\lambda$ is a scalar, is equivalent to

$$
\hat{\mathbf{P}}_{u} \mathbf{A}_{u}+\mathbf{A}_{u}^{T} \hat{\mathbf{P}}_{u}=\hat{\mathbf{P}}_{u} \mathbf{B}_{u} \lambda^{-1} \mathbf{B}_{u}^{T} \lambda \hat{\mathbf{P}}_{u} .
$$

Now multiply both sides of the above expression by $\lambda$ and define $\hat{\mathbf{P}}_{u}^{\lambda}=\lambda \hat{\mathbf{P}}_{u}$

$$
\hat{\mathbf{P}}_{u}^{\lambda} \mathbf{A}_{u}+\mathbf{A}_{u}^{T} \hat{\mathbf{P}}_{u}^{\lambda}=\hat{\mathbf{P}}_{u}^{\lambda} \mathbf{B}_{u} \lambda^{-1} \mathbf{B}_{u}^{T} \hat{\mathbf{P}}_{u}^{\lambda},
$$

and thus $\hat{\mathbf{P}}_{u}^{\lambda}$ satisfies (3) with $R=\lambda$, and thus $\hat{\mathbf{P}}^{\lambda}$ satisfies (2) with $R=\lambda$, which concludes the proof.

Corollary 2: (Transformed Closed-Form Solution) Consider a nonsingular transformation of the state $\mathbf{T}$

$$
\mathbf{T}=\left[\begin{array}{ll}
\mathbf{T}_{1} & \mathbf{T}_{2} \\
\mathbf{T}_{3} & \mathbf{T}_{4}
\end{array}\right]
$$

with $\mathbf{T}_{1} \in \mathbb{R}^{m \times m}, \mathbf{T}_{2} \in \mathbb{R}^{m \times n-m}, \mathbf{T}_{3} \in \mathbb{R}^{n-m \times m}$ and $\mathbf{T}_{4} \in \mathbb{R}^{n-m \times n-m}$. The closed-form solution to the minimum energy ARE in (2) with weight $R=1$ in the transformed state $\bar{x}=T x$, is given by

$$
\overline{\mathbf{P}}=\left[\begin{array}{c}
\mathbf{T}_{1}^{T} \\
\mathbf{T}_{2}^{T}
\end{array}\right] \hat{\mathbf{P}}_{u}\left[\begin{array}{ll}
\mathbf{T}_{1} & \mathbf{T}_{2}
\end{array}\right],
$$

with $\hat{\mathbf{P}}_{u}$ as in (5).

Proof: Subject to a nonsingular transformation $\mathbf{T}$ the new matrices $\overline{\mathbf{A}}$ and $\overline{\mathbf{B}}$ are given by $\mathbf{T}^{-1} \mathbf{A T}$ and $\mathbf{T}^{-1} \mathbf{B}$ respectively. Consider now Proposition 1 and the minimum energy ARE in (2) with $R=1$ solved by $\mathbf{P}=\hat{\mathbf{P}}$. Multiply both sides by $\mathbf{T}$ from the right and by $\mathbf{T}^{T}$ from the left

$$
\mathbf{T}^{T} \hat{\mathbf{P}} \mathbf{A T}+\mathbf{T}^{T} \mathbf{A}^{T} \hat{\mathbf{P}} \mathbf{T}=\mathbf{T}^{T} \hat{\mathbf{P B B}} \mathbf{B}^{T} \hat{\mathbf{P}} \mathbf{T} .
$$

Since $\mathbf{T}$ is nonsingular we can define the change $\mathbf{T}^{-T} \overline{\mathbf{P}} \mathbf{T}^{-1}=\hat{\mathbf{P}}$. Replace in the above expression and rearrange terms according to the properties of transposition to obtain

$$
\overline{\mathbf{P}} \overline{\mathbf{A}}+\overline{\mathbf{A}}^{T} \overline{\mathbf{P}}=\overline{\mathbf{P}} \overline{\mathbf{B}} \overline{\mathbf{B}}^{T} \mathbf{P} .
$$

Thus $\overline{\mathbf{P}}$ solves the "transformed" minimum energy ARE. Replacing $\mathbf{T}$ as in (7) and $\hat{\mathbf{P}}$ as in (4) gives $\overline{\mathbf{P}}$ as in (8) which concludes the proof.

\section{DISCUSSION}

A. Numerical Examples and Numerical Comparison with the Matlab Solution

Example 1: Consider the plant model $G(s)=$ $\frac{(s+0.3)}{(s+1)(s-2)(s-7)}$. We recognise $G_{u}(s)$ and $G_{s}(s)$ as

$$
G_{u}(s)=\frac{0.0292(s+24.25)}{(s-2)(s-7)}, \quad G_{s}(s)=\frac{-0.0292}{s+1} .
$$

From imposing $\mathbf{A}_{u}$ and $\mathbf{B}_{u}$ as in assumption 3) we have

$$
\mathbf{A}_{u}=\left[\begin{array}{ll}
2 & 0 \\
0 & 7
\end{array}\right], \quad \mathbf{B}_{u}=\left[\begin{array}{l}
1 \\
1
\end{array}\right]
$$

and finally from the partial fraction expansion of $G_{u}(s)$

$$
G_{u}(s)=\frac{-0.1533}{(s-2)}+\frac{0.1825}{(s-7)},
$$

we obtain $\mathbf{C}_{u}$ as $[-0.15330 .1825]$. We then recognise $p_{1}=2$ and $p_{2}=7$. From Proposition 1 we have that

$$
\begin{aligned}
& r_{1}=2 p_{1}\left(\frac{p_{1}+p_{2}}{p_{1}-p_{2}}\right)=-7.2 \\
& r_{2}=2 p_{2}\left(\frac{p_{2}+p_{1}}{p_{2}-p_{1}}\right)=25.2 .
\end{aligned}
$$


The closed-form solution $\hat{\mathbf{P}}_{u}$ for this example is then given by

$$
\hat{\mathbf{P}}_{u}=\left[\begin{array}{cc}
\frac{r_{1}^{2}}{2 p_{1}} & \frac{r_{1} r_{2}}{p_{1}+p_{2}} \\
\frac{r_{2} r_{1}}{p_{2}+p_{1}} & \frac{r_{2}^{2}}{2 p_{2}}
\end{array}\right]=\left[\begin{array}{cc}
12.96 & -20.16 \\
-20.16 & 45.36
\end{array}\right]
$$

and the overall solution $\hat{\mathbf{P}}$ is then given by

$$
\hat{\mathbf{P}}=\left[\begin{array}{ccc}
12.96 & -20.16 & 0 \\
-20.16 & 45.36 & 0 \\
0 & 0 & 0
\end{array}\right]
$$

We compare next another example of a direct application of Proposition 1 to its numerical solution obtained with Matlab.

Example 2: Consider in this example $\mathbf{A}_{u}$ and $\mathbf{B}_{u}$ as

$$
\mathbf{A}_{u}=\left[\begin{array}{cccc}
p_{1} & 0 & 0 & 0 \\
0 & p_{2} & 0 & 0 \\
0 & 0 & p_{3} & 0 \\
0 & 0 & 0 & p_{4}
\end{array}\right], \quad \mathbf{B}_{u}=\left[\begin{array}{l}
1 \\
1 \\
1 \\
1
\end{array}\right],
$$

where $\left.\left.p_{1} \in\right] 0,15\right], p_{2}=\sqrt{2}, p_{3}=\sqrt{5}$ and $p_{4}=\sqrt{7}$. The closed-form solution $\mathbf{P}_{u}$ for the minimum energy ARE (3) for this example is given by

$$
\hat{\mathbf{P}}_{u}=\left[\begin{array}{cccc}
\frac{r_{1}^{2}}{2 p_{1}} & \frac{r_{1} r_{2}}{p_{1}+p_{2}} & \frac{r_{1} r_{3}}{p_{1}+p_{3}} & \frac{r_{1} r_{4}}{p_{1}+p_{4}} \\
\frac{r_{2} r_{1}}{p_{2}+p_{1}} & \frac{r_{2}^{2}}{2 p_{2}} & \frac{r_{2} r_{3}}{p_{2}+p_{3}} & \frac{r_{2} r_{4}}{p_{2}+p_{4}} \\
\frac{r_{3} r_{1}}{p_{3}+p_{1}} & \frac{r_{3} r_{2}}{p_{3}+p_{2}} & \frac{r_{3}^{2}}{2 p_{3}} & \frac{r_{3} r_{4}}{p_{3}+p_{4}} \\
\frac{r_{4} r_{1}}{p_{4}+p_{1}} & \frac{r_{4} r_{2}}{p_{4}+p_{2}} & \frac{r_{4} r_{3}}{p_{4}+p_{3}} & \frac{r_{4}^{2}}{2 p_{4}}
\end{array}\right],
$$

with

$$
\begin{aligned}
& r_{1}=2 p_{1} \frac{\left(p_{1}+p_{2}\right)\left(p_{1}+p_{3}\right)\left(p_{1}+p_{4}\right)}{\left(p_{1}-p_{2}\right)\left(p_{1}-p_{3}\right)\left(p_{1}-p_{4}\right)}, \\
& r_{2}=2 p_{2} \frac{\left(p_{2}+p_{1}\right)\left(p_{2}+p_{3}\right)\left(p_{2}+p_{4}\right)}{\left(p_{2}-p_{1}\right)\left(p_{2}-p_{3}\right)\left(p_{2}-p_{4}\right)}, \\
& r_{3}=2 p_{3} \frac{\left(p_{3}+p_{1}\right)\left(p_{3}+p_{2}\right)\left(p_{3}+p_{4}\right)}{\left(p_{3}-p_{1}\right)\left(p_{3}-p_{2}\right)\left(p_{3}-p_{4}\right)}, \\
& r_{4}=2 p_{4} \frac{\left(p_{4}+p_{1}\right)\left(p_{4}+p_{2}\right)\left(p_{4}+p_{3}\right)}{\left(p_{4}-p_{1}\right)\left(p_{4}-p_{2}\right)\left(p_{4}-p_{3}\right)} .
\end{aligned}
$$

We compare the expression in (11) with the solution resulting from the Matlab command care, based on [12], by executing the line

$$
\mathbf{P}_{u}^{m}=\operatorname{care}\left(\mathrm{A}_{\mathrm{u}}, \mathrm{B}_{\mathrm{u}}, \operatorname{zeros}(4), 1\right),
$$

in Matlab (7.5.0.342 (R2007b)) with $\mathbf{A}_{u}$ and $\mathbf{B}_{u}$ as in (10). To quantify the difference between the closed-form solution and the Matlab solution, we propose the following error function

$$
e_{A R E}=\sum_{\forall i, j}\left[\mathbf{P}_{u} \mathbf{A}_{u}+\mathbf{A}_{u}^{T} \mathbf{P}_{u}-\mathbf{P}_{u} \mathbf{B}_{u} \mathbf{B}_{u}^{T} \mathbf{P}_{u}\right]_{i, j}^{2},
$$

either with $\mathbf{P}_{u}=\hat{\mathbf{P}}_{u}$ or $\mathbf{P}_{u}=\mathbf{P}_{u}^{m}$. Notice that $e_{A R E}$ is defined as to quantify the square difference between the LHS and RHS of (3), that is our proposed error function is quantifying the precision of each $\mathbf{P}_{u}$ solution, either in closed-form or from Matlab. The result for $e_{A R E}$ can be observed in Figure 2, where the solid line is $e_{A R E}$ obtained with the solution using Matlab, whilst the dashed line is
$e_{A R E}$ obtained with $\hat{\mathbf{P}}_{u}$. For both approaches, either closedform or Matlab, we observe how for all values of $p_{1}$ the error is indeed very small, in the order of $-200(\mathrm{~dB})$ (where $(\mathrm{dB}) \mathrm{s}$ are obtained as $10 \log _{10} e_{A R E}$ accounting for the squared definition of the error function $e_{A R E}$ ). Also as $p_{1}$ approaches $p_{2}, p_{3}$ or $p_{4}$ the error tends to grow. Indeed the higher error value located in Figure 2 at $p_{2}, p_{3}$ and $p_{4}$ is signalling the loss of controllability that occurs when $p_{1}$ matches any of these values. In the neighbourhood of such values the quasiloss of controllability produces $\mathbf{P}_{u}$ solutions with very high entries in each of its elements making numerical errors all the more significant. The higher value in each of the entries of $\mathbf{P}_{u}$ can also be observed from the expressions for $r_{1}, r_{2}$, $r_{3}$ and $r_{4}$ in (12). Whenever $p_{1}$ approaches the values of the other unstable eigenvalues, then each $r_{i}$ will grow due to the factor $p_{1}-p_{i}$ (with $i=2,3,4$ ) in each of their denominators. The error at precisely the value of $p_{2}, p_{3}$ and $p_{4}$ should grow to infinity (as the closed-form suggests), but this is not the case in Figure 2 due to interpolation.

\section{B. Theoretical Comparison with the Matlab Solution}

The comparison of the closed-form solution $\hat{\mathbf{P}}_{u}$ and the one obtained with Matlab in the previous example calls for a closer comparison with the ideas behind the algorithm implemented in Matlab. From [12] we observe that the command care, for the proposed class of minimum energy AREs, solves an eigenproblem defined by the symplectic matrix

$$
\mathbf{M}=\left[\begin{array}{cc}
\mathbf{A}_{u} & -\mathbf{B}_{\mathbf{u}} R^{-1} \mathbf{B}_{\mathbf{u}}^{T} \\
0 & -\mathbf{A}_{u}^{T}
\end{array}\right] .
$$

The solution to the eigenproblem $\mathbf{W}$ is such that we have

$$
\mathbf{W}^{-1} \mathbf{M W}=\left[\begin{array}{cc}
-\mathbf{A}_{u} & \mathbf{0} \\
\mathbf{0} & \mathbf{A}_{u}
\end{array}\right],
$$

and $\mathbf{P}_{u}=\mathbf{W}_{21} \mathbf{W}_{11}^{-1}$, see for example [13, §15.3]. Define then, without loss of generality, $\mathbf{W}$ to be

$$
\mathbf{W}=\left[\begin{array}{cc}
\mathbf{P}_{u}^{-1} & \mathbf{I} \\
\mathbf{I} & \mathbf{0}
\end{array}\right]
$$

and its inverse the matrix

$$
\mathbf{W}^{-1}=\left[\begin{array}{cc}
\mathbf{0} & \mathbf{I} \\
\mathbf{I} & -\mathbf{P}_{u}^{-1}
\end{array}\right]
$$

where we have invoked the Woodbury Matrix Identity, see for example [14, p.502]. We can observe then that in order to satisfy (13) the following expression needs to hold

$$
\mathbf{A}_{u} \mathbf{P}_{u}^{-1}-\mathbf{B}_{u} R^{-1} \mathbf{B}_{u}^{T}+\mathbf{P}_{u}^{-1} \mathbf{A}_{u}^{T}=\mathbf{0},
$$

which indeed is the case, since by multiplying from the right and from the left by $\mathbf{P}_{u}$ we retrieve (3). Thus, as intuition would have it, from Proposition 1 we can also obtain a closed-form solution to the eigenproblem solved by the algorithm proposed in [12], that is for $R=1$ we have

$$
\hat{\mathbf{W}}=\left[\begin{array}{cc}
\hat{\mathbf{P}}_{u}^{-1} & \mathbf{I} \\
\mathbf{I} & \mathbf{0}
\end{array}\right]
$$




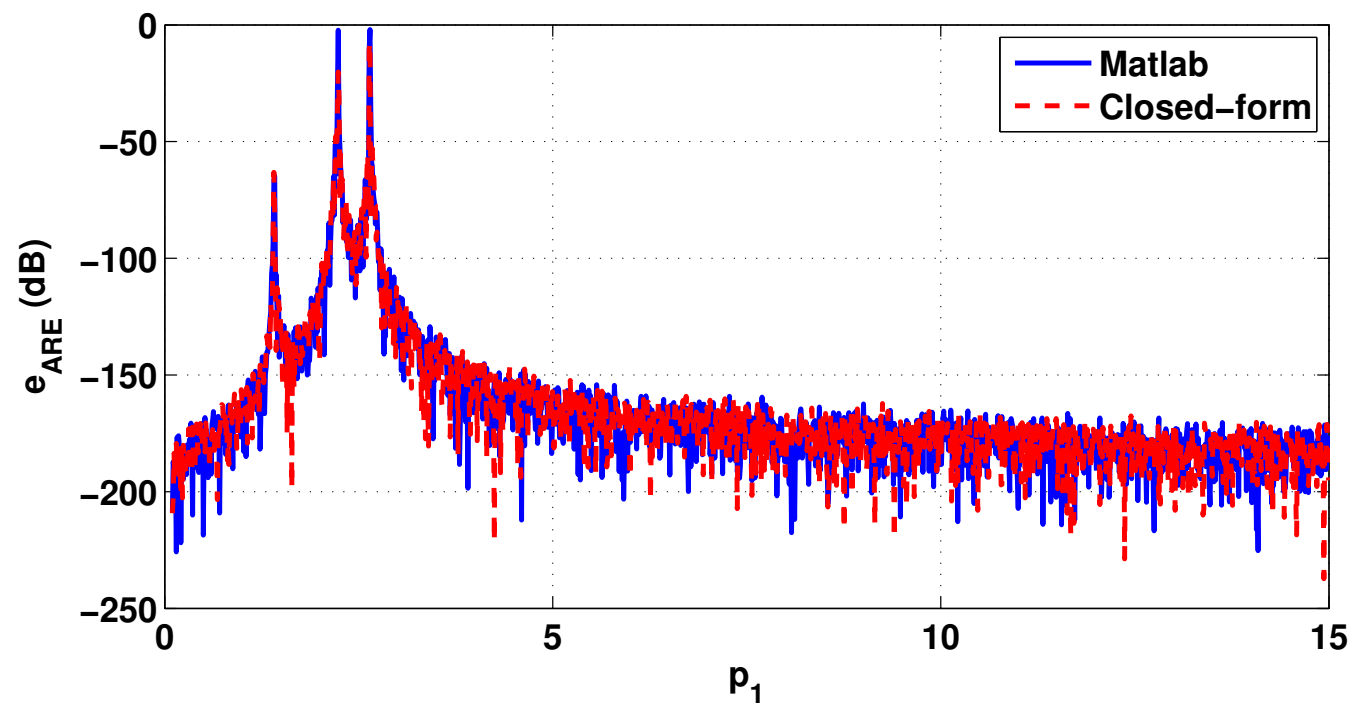

Fig. 2. Numerical error $e_{A R E}$ upon replacing $\mathbf{P}_{u}$ obtained from Matlab, solid line, or in closed-form, dashed line.

and for $R=\lambda$ we have

$$
\hat{\mathbf{W}}^{\lambda}=\left[\begin{array}{cc}
\left(\hat{\mathbf{P}}_{u}^{\lambda}\right)^{-1} & \mathbf{I} \\
\mathbf{I} & \mathbf{0}
\end{array}\right]
$$

Remark 1: Notice that equation (14) is a Lyapunov equation and as such a closed-form solution for $\mathbf{P}_{u}^{-1}$ in (14) can easily be found independently of Proposition 1. Nonetheless, we point out that in general such closed-form solution for $\mathbf{P}_{u}^{-1}$ does not imply directly a closed-form for $\hat{\mathbf{P}}_{u}$ (such as the one obtained in Proposition 1).

\section{Repeated Poles Extension of the Main Result}

Finally, the study of repeated poles is also of interest, but it is also more complex. Nonetheless as an example we present next the case of a pair of repeated eigenvalues.

Example 3: We treat in the present example the case that $\mathbf{A}_{u}$ contains a pair of repeated eigenvalues in $\mathbb{R}^{+}$, that is we have

$$
\mathbf{A}_{u}=\left[\begin{array}{cc}
p_{1} & 1 \\
0 & p_{1}
\end{array}\right], \quad \mathbf{B}_{u}=\left[\begin{array}{l}
0 \\
1
\end{array}\right] .
$$

The closed-form solution of the minimum energy Riccati equation (3), with $\mathbf{A}_{u}$ and $\mathbf{B}_{u}$ as above, is given by

$$
\hat{\mathbf{P}}_{u}=\left[\begin{array}{ll}
8 p_{1}^{3} & 4 p_{1}^{2} \\
4 p_{1}^{2} & 4 p_{1}
\end{array}\right]
$$

We can confirm this by directly replacing the above solution in (3), with $\mathbf{A}_{u}$ and $\mathbf{B}_{u}$ as in (15) and $R=1$.

The above example illustrates how hard it can be, when considering repeated unstable eigenvalues, to gain sufficient insight into the minimum energy problem as to find $\hat{\mathbf{P}}_{u}$ in closed-form. At the very least, as a first step to pursue such extension, there is the need to extend [7, Theorem 1] to repeated unstable eigenvalues.

\section{CONCLUSION}

In the present paper we have obtained a continuous-time closed-form solution for a class of Minimum Energy algebraic Riccati equations. This particular class is characterised by Riccati equations which consider only distinct eigenvalues for the spectrum of $\mathbf{A}_{u}$ and a vanishing state weight. As an example we have compared the closed-form result to the one obtained using the command care in Matlab, both numerically and theoretically. Future research will consider lifting the condition of distinct eigenvalues for $\mathbf{A}_{u}$, as well as the study of multiple input multiple output systems and the dual discrete-time minimum energy ARE case.

\section{APPENDIX}

We present here two lemmas required in the proof of Proposition 1.

Lemma 1: the following equality holds

$$
\begin{aligned}
& \sum_{j=1}^{k_{o}} \frac{2 p_{k_{o}+1}}{p_{i}+p_{j}} \frac{q_{j}}{p_{j}-p_{k_{o}+1}}+\frac{t_{k_{o}+1}}{p_{i}+p_{k_{o}+1}}=0, \quad \forall i=1, \cdots, k_{o}, \\
& \text { where } \quad q_{j}=2 p_{j} \prod_{\substack{l=1 \\
l \neq j}}^{k_{o}} \frac{p_{j}+p_{l}}{p_{j}-p_{l}} \quad \text { and } t_{k_{o}+1}= \\
& 2 p_{k_{o}+1} \prod_{l=1}^{k_{o}} \frac{p_{k_{o}+1}+p_{l}}{p_{k_{o}+1}-p_{l}} \text {. } \\
& \quad \text { Proof: observe that }(16) \text { can be rewritten as }
\end{aligned}
$$

$$
2 p_{k_{o}+1} \sum_{j=1}^{k_{o}} \frac{1}{p_{i}+p_{j}} \frac{q_{j}}{p_{j}-p_{k_{o}+1}}+\frac{2 p_{k_{o}+1} \prod_{l=1}^{k_{o}} \frac{p_{k_{o}+1}+p_{l}}{p_{k_{o}+1}-p_{l}}}{p_{i}+p_{k_{o}+1}} .
$$


ThBIn3.10

More so the term $\frac{\prod_{l=1}^{k_{o}} \frac{p_{k_{o}}+p_{l}}{p_{k_{o}}-p_{l}}}{p_{i}+p_{k_{o}+1}}$ can be decomposed in a partial fraction expansion such as

$$
\begin{aligned}
& \frac{\prod_{l=1}^{k_{o}} \frac{p_{k_{o}+1}+p_{l}}{p_{k_{o}+1}-p_{l}}}{p_{i}+p_{k_{o}+1}}=\frac{\prod_{\substack{l=1 \\
l \neq i}}^{k_{o}} p_{k_{o}+1}+p_{l}}{\prod_{l=1}^{k_{o}} p_{k_{o}+1}-p_{l}} \\
&=\sum_{l=1}^{k_{o}} \frac{1}{p_{i}+p_{l}} \frac{q_{l}}{p_{k_{o}+1}-p_{l}},
\end{aligned}
$$

which replaced in (17) gives

$$
\begin{aligned}
2 p_{k_{o}+1} \sum_{j=1}^{k_{o}} \frac{1}{p_{i}+p_{j}} & \frac{q_{j}}{p_{j}-p_{k_{o}+1}} \\
& +2 p_{k_{o}+1} \sum_{l=1}^{k_{o}} \frac{1}{p_{i}+p_{l}} \frac{q_{l}}{p_{k_{o}+1}-p_{l}},
\end{aligned}
$$

and thus

$$
\begin{aligned}
& 2 p_{k_{o}+1} \sum_{j=1}^{k_{o}} \frac{1}{p_{i}+p_{j}} \frac{q_{j}}{p_{j}-p_{k_{o}+1}} \\
&-2 p_{k_{o}+1} \sum_{l=1}^{k_{o}} \frac{1}{p_{i}+p_{l}} \frac{q_{l}}{p_{l}-p_{k_{o}+1}}=0,
\end{aligned}
$$

which ends the proof.

Lemma 2: assume $r_{i}$ as in (6), then the following equality holds

$$
\sum_{j=1}^{m} \frac{r_{j}}{p_{i}+p_{j}}=1, \quad \forall i=1, \cdots, m
$$

Proof: the following proof is based on an induction argument:

1) For $m=1$ we have $2 p_{1} / 2 p_{1}=1$.

2) Assume the case $m=k_{o}$

$$
\sum_{j=1}^{k_{o}} \frac{q_{j}}{p_{i}+p_{j}}=1, \quad \forall i=1, \cdots, m,
$$

to be true for any $i=1, \cdots, k_{o}$ with $q_{j}=$ $2 p_{j} \prod_{\substack{l=1 \\ l \neq j}}^{k_{o}} \frac{p_{j}+p_{l}}{p_{j}-p_{l}}$.

3) To prove the case $m=k_{o}+1$ start from (18) and observe that by applying Lemma 1 we have $\forall i=$ $1, \cdots, k_{o}$, that

$\sum_{j=1}^{k_{o}} \frac{q_{j}}{p_{i}+p_{j}}+\sum_{j=1}^{k_{o}} \frac{2 p_{k_{o}+1}}{p_{i}+p_{j}} \frac{q_{j}}{p_{j}-p_{k_{o}+1}}+\frac{t_{k_{o}+1}}{p_{i}+p_{k_{o}+1}}=1$,

where $t_{k_{o}+1}=2 p_{k_{o}+1} \prod_{i=1}^{k_{o}} \frac{p_{k_{o}+1}+p_{i}}{p_{k_{o}+1}-p_{i}}$ and in general $t_{j}=2 p_{j} \prod_{\substack{l=1 \\ l \neq j}}^{k_{o}+1} \frac{p_{j}+p_{l}}{p_{j}-p_{l}}$. Rearrange terms to observe that

$$
\sum_{j=1}^{k_{o}}\left(1+\frac{2 p_{k_{o}+1}}{p_{j}-p_{k_{o}+1}}\right) \frac{q_{j}}{p_{i}+p_{j}}+\frac{t_{k_{o}+1}}{p_{i}+p_{k_{o}+1}}=1,
$$

thus

$$
\begin{aligned}
\sum_{j=1}^{k_{o}}\left(\frac{p_{j}+p_{k_{o}+1}}{p_{j}-p_{k_{o}+1}}\right) & \frac{q_{j}}{p_{i}+p_{j}}+\frac{t_{k_{o}+1}}{p_{i}+p_{k_{o}+1}} \\
& =\sum_{j=1}^{k_{o}} \frac{t_{j}}{p_{i}+p_{j}}+\frac{t_{k_{o}+1}}{p_{i}+p_{k_{o}+1}},
\end{aligned}
$$

and

$$
\sum_{j=1}^{k_{o}+1} \frac{t_{j}}{p_{i}+p_{j}}=1, \quad \forall i=1, \cdots, k_{o} .
$$

4) Finally for the case of $m=k_{o}+1$ and $i=k_{o}+1$ consider the following development

$$
\begin{aligned}
& \sum_{j=1}^{k_{o}+1} \frac{r_{j}}{p_{k_{o}+1}+p_{j}} \\
& =\sum_{j=1}^{k_{o}} \frac{r_{j}}{p_{k_{o}+1}+p_{j}}+\frac{r_{k_{o}+1}}{p_{k_{o}+1}+p_{k_{o}+1}} \\
& =\sum_{j=1}^{k_{o}} \frac{q_{j}}{p_{j}-p_{k_{o}+1}}+\prod_{l=1}^{k_{o}} \frac{p_{k_{o}+1}+p_{l}}{p_{k_{o}+1}-p_{l}} \\
& =\sum_{j=1}^{k_{o}} \frac{q_{j}}{p_{j}-p_{k_{o}+1}}+\sum_{j=1}^{k_{o}} \frac{q_{j}}{p_{k_{o}+1}-p_{j}}+1=1,
\end{aligned}
$$

which ends the proof.

\section{REFERENCES}

[1] W.T. Reid. Riccati Differential Equations. Academic Press, 1972.

[2] P. Lancaster and L. Rodman. Algebraic Riccati Equations. Oxford Science Publications, 1995.

[3] G.C. Goodwin, S.F. Graebe, and M.E. Salgado. Control System Design. Prentice Hall, 2001.

[4] Special Issue on Networked Control Systems. IEEE Transactions on Automatic Control, 49(9), September 2004.

[5] G.N. Nair, F Fagnani, S Zampieri, and R.J. Evans. Feedback Control under Data Rate Constraints: an Overview. Proceedings of the IEEE (special issue on "The Emerging Technology of Networked Control Systems"), 95(1):108-137, January 2007.

[6] J.H. Braslavsky, R.H. Middleton, and J.S. Freudenberg. Feedback Stabilisation over Signal-to-Noise Ratio Constrained Channels. IEEE Transactions on Automatic Control, 52(8):1391-1403, 2007.

[7] A.J. Rojas, J.H. Braslavsky, and R.H. Middleton. Output Feedback Stabilisation over Bandwidth Limited, Signal to Noise Ratio Constrained Communication Channels. In Proceedings of the 2006 American Control Conference, Minneapolis, USA, pages 2789-2794, June 2006.

[8] B. Bassam and P.G. Voulgaris. A convex characterization of distributed control problems in spatially invariant systems with communication constraints. System and \& Control Letters, 54:574-583, 2005.

[9] A. Rantzer. Linear quadratic team theory revisited. In Proceedings of the 2006 American Control Conference, pages 1637-1641, Minneapolis, USA, June 2006.

[10] A.J. Rojas. Closed-form solution for a class of discrete-time algebraic riccati equations. In Proceedings of the 2009 American Control Conference, pages 482-487, St. Louis, Missouri, USA, June 2009.

[11] J.H. Braslavsky, M.M. Seron, D.Q. Mayne, and P.V. Kokotovic. Limiting performance of optimal filters. Automatica, 35(2):pp. 189199, February 1999.

[12] W.F.III Arnold and A.J. Laub. Generalized Eigenproblem Algorithms and Software for Algebraic Riccati Equations. Proceedings of the IEEE, 72(12):1746-1754, December 1984

[13] B. Anderson and J. Moore. Linear Optimal Control. Prentice Hall International, 1989.

[14] E.W. Grafarend. Linear and Nonlinear Models: Fixed Effects, Random Effects, and Mixed Models. Walter de Gruyter, 2006. 